\title{
Carcinoma associated fibroblasts derived from oral squamous cell carcinoma promote lymphangiogenesis via c-Met/PI3K/AKT in vitro
}

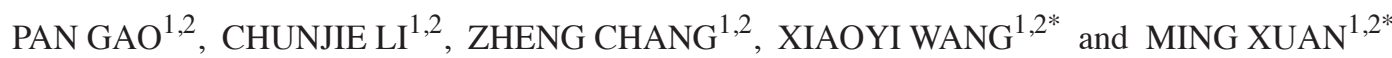 \\ ${ }^{1}$ State Key Laboratory of Oral Diseases; ${ }^{2}$ Department of Head and Neck Oncology, West China Hospital of Stomatology, \\ Sichuan University, Chengdu, Sichuan 610041, P.R. China
}

Received December 26, 2016; Accepted September 7, 2017

DOI: $10.3892 / \mathrm{ol} .2017 .7301$

\begin{abstract}
Carcinoma-associated fibroblasts (CAFs) are dominant components of the tumor microenvironment (TME) that promote the development, progression and metastasis of cancer. c-Met is a receptor of the hepatocyte growth factor (HGF), which is involved in lymphangiogenesis. Currently, the roles of CAFs during lymphangiogenesis are unknown. It has been hypothesized that CAFs contribute to lymphangiogenesis of oral squamous cell carcinoma (OSCC) via a HGF/c-Met complex. The expression of HGF in OSCC was determined using CAFs derived from OSCC tissue and it was demonstrated that HGF is overexpressed in OSCC-derived CAFs. It was also revealed that c-Met was highly expressed in human lymphatic endothelial cells (HLECs) when co-cultured with CAFs. Furthermore, it was demonstrated that recombinant human HGF significantly enhanced the proliferation, migration, invasion and tube formation of HLECs. By contrast, the inhibition of c-Met expression suppressed the aforementioned biological activities and also downregulated the expression of c-Met, phosphoinositide 3-kinase and phosphorylated protein kinase B. Taken together, these data demonstrate that c-Met is associated with the regulation of lymphangiogenesis. Thus, the results of the present study indicate that c-Met may be a promising novel therapeutic target to treat patients with OSCC.
\end{abstract}

Correspondence to: Professor Xiaoyi Wang or Professor Ming Xuan, Department of Head and Neck Oncology, West China Hospital of Stomatology, Sichuan University, 14 Third Section, Renmin Nan Road, Chengdu, Sichuan 610041, P.R. China

E-mail:wxyz711@163.com

E-mail:2377234799@qq.com

*Contributed equally

Key words: oral squamous cell carcinoma, carcinoma-associated fibroblasts, hepatocyte growth factor, c-Met, lymphangiogenesis

\section{Introduction}

Metastasis to the regional lymph nodes is a major factor indicating the negative prognosis of patients with cancer. Whether or not metastasis has occurred influences whether a patient receives postoperative therapy, including chemo- and radiotherapy, to reduce the risk of metastatic recurrence (1). Carcinoma-induced lymphangiogenesis within the tumor tissue is one of the causes of lymph node metastasis and it has been revealed that stromal and cancer cells serve a critical role in the induction of lymphangiogenesis by secreting lymphangiogenic factors, including vascular endothelial growth factor (VEGF) and platelet-derived growth factor (PDGF) (2). Carcinoma-associated fibroblasts (CAFs) are the most abundant stromal cells in the tumor microenvironment (TME) and promote tumor growth, angiogenesis and the malignant progression of various solid tumors (3). In addition, CAFs use the epithelial-mesenchymal transition, a major biological process that can stimulate cancer progression and metastasis, to regulate the invasiveness of tumor cells $(4,5)$.

The hepatocyte growth factor (HGF) receptor c-Met is encoded by the proto-oncogene MET. MET exhibits tyrosine-protein kinase activity and is overexpressed in numerous types of cancer, such as head and neck squamous cell carcinoma (6,7). Furthermore, the HGF/c-MET complex is widely expressed in cells of epithelial-endothelial origin and regulates multiple cellular processes in cancer, including cell proliferation, invasion and angiogenesis (7). Although a correlation between CAFs and cancer progression and metastasis has been determined $(4,5)$, it remains unknown whether CAFs contribute to lymphangiogenesis in oral squamous cell carcinoma (OSCC) via the HGF/c-Met complex. Therefore, identifying the complex interactions between CAFs and lymphatic endothelial cells may help to identify the underlying mechanisms of CAFs in the lymphangiogenesis of OSCC.

CAFs and normal fibroblasts (NFs) have been successfully isolated and characterized from OSCC as well as from normal oral mucosal tissues (8). A co-culture experiment in a Transwell system revealed that CAFs significantly promote the proliferation, migration, invasion and tube formation of human lymphatic endothelial cells (HLECs) (8). The present study used a chemiluminescent immunoassay (CLIA) to measure 
the secretion of HGF by CAFs and to evaluate the expression of c-Met in HLECs co-cultured with CAFs. Furthermore, the influence of HGF on the proliferation, migration, invasion and tube formation of HLEC was evaluated using recombinant human HGF (rhHGF). An inhibitor of c-Met, JNJ-38877605, suppressed the proliferation, migration and invasion, as well as the tube formation, of HLECs by downregulating phosphatidylinositol 3-kinase (PI3K) and phosphorylated protein kinase B (AKT) levels. Thus, the results of the current study indicate that the c-Met/PI3K/AKT signaling pathway is one mechanism by which CAFs regulate lymphangiogenesis.

\section{Materials and methods}

Cell culture. The present study was approved by the Medical Ethics Committee of the West China Hospital of Stomatology of Sichuan University (Chengdu, China). Specimens of OSCC and normal oral mucosal tissue were obtained from 5 patients (2 males, 3 females; mean age, 61.6 years) from November 2015 to December 2015 at the Department of Head and Neck Oncology, West China Hospital of Stomatology, and all patients provided informed consent prior to inclusion in the current study. The isolation and characterization of primary CAFs and NFs was performed according to a previously established protocol (8). CAFs and NFs were cultured in high-glucose Dulbecco's modified Eagle's medium (DMEM; GE Healthcare, Chicago, IL, USA) containing 10\% fetal bovine serum (FBS; Thermo Fisher Scientific, Inc., Waltham, MA, USA), penicillin (100 U/ml) and streptomycin $(100 \mu \mathrm{g} / \mathrm{ml}$; GE Healthcare) at $37^{\circ} \mathrm{C}$ in a humidified incubator with $5 \% \mathrm{CO}_{2}$. HLECs were purchased from ScienCell Research Laboratories (Carlsbad, CA, USA) and cultured in endothelial cell medium (ECM; ScienCell Research Laboratories) supplemented with endothelial cell growth supplement (ScienCell Research Laboratories), $10 \%$ FBS, penicillin $(100 \mathrm{U} / \mathrm{ml})$ and streptomycin $(100 \mu \mathrm{g} / \mathrm{ml})$ at $37^{\circ} \mathrm{C}$ in a $5 \% \mathrm{CO}_{2}$ humidified atmosphere. Cell culture duration is described in each specific experiment as follows.

CLIA. CAFs and NFs (third passage, $2 \times 10^{5}$ cells/well) were plated in 6-well plates in high-glucose DMEM containing $10 \%$ FBS. When cells reached $60-70 \%$ confluence, they were incubated further at $37^{\circ} \mathrm{C}$ in FBS-free DMEM-high glucose for $24 \mathrm{~h}$. Culture medium were collected and centrifuged at $1,000 \mathrm{x} \mathrm{g}$, at $4^{\circ} \mathrm{C}$ for $20 \mathrm{~min}$ and the supernatant was evaluated using an HGF CLIA kit (USCN Life Science, Inc., Wuhan, China) following the manufacturer's protocol.

Cell proliferation assay. Cell Counting kit-8 (CCK-8) assays (Dojindo Molecular Technologies, Inc., Kumamoto, Japan) were used to evaluate the proliferation of HLECs. HLECs induced by rhHGF (PeproTech, Inc., Rocky Hill, NJ, USA) or JNJ-38877605 (400 nM; Selleck Chemicals, Houston, TX, USA) were seeded in 96-well plates $(2,000$ cells/well, $100 \mu \mathrm{l})$ and incubated at $37^{\circ} \mathrm{C}$ for 12 or $24 \mathrm{~h}$. For rhHGF, the blank control was the culture medium without cells. The negative control used the vehicle, deionized water for rhHGF and DMSO for JNJ-38877605. Cells were incubated with $10 \mu \mathrm{l}$ CCK- 8 for $2 \mathrm{~h}$, and optical density (OD) was, measured at $450 \mathrm{~nm}$ using Varioskan Flash microplate reader (Thermo Fisher Scientific, Inc.).
Cell migration and invasion assays. HLEC migration and invasion assays were performed in a 24-well Transwell system (8 $\mu \mathrm{m}$ pore size; Corning, Corning, NY, USA). In these assays, rhHGF, CAFs or CAFs and JNJ-38877605 (400 nM) were plated in the lower chamber. HLECs $\left(3 \times 10^{5}\right.$ cells in $300 \mu \mathrm{l}$ medium) were seeded in the upper chamber coated with Matrigel (BD Biosciences, San Jose, CA, USA) for the invasion assay. The same protocol was used to carry out the migration assay except without coating Matrigel in the upper chamber. Deionized water and CAFs without JNJ-38877605 were taken as the respective negative control in the two assays. Following incubation for $24 \mathrm{~h}$, the non-penetrating cells on the upper surface of the filters were removed using a cotton swab. The migrated and invaded cells were fixed in $4 \%$ paraformaldehyde for $5 \mathrm{~min}$ at room temperature and stained with $0.1 \%$ crystal violet for $15 \mathrm{~min}$. Following each step, filters were washed 3 times in PBS. Three random fields were selected and the cells on each filter were photographed using an inverted phase contrast microscope (Olympus Corporation, Tokyo, Japan) at a magnification of $\mathrm{x} 100$.

Matrigel in vitro HLEC tube formation assay. The Matrigel in vitro assay was used to evaluate HLEC tube formation following rhHGF treatment or co-culture with CAFs that were supplemented with JNJ-38877605 or not. Matrigel was plated at the bottom of a 24-well plate or the lower chamber of the 24-well Transwell system $(0.4 \mu \mathrm{m}$; Corning) following thawing overnight at $4^{\circ} \mathrm{C}$ and then kept at $37^{\circ} \mathrm{C}$ for $60 \mathrm{~min}$. HLEC cells $\left(1 \times 10^{5}\right)$ in $500 \mu \mathrm{l} \mathrm{ECM}$ containing $5 \% \mathrm{FBS}$ and endothelial cell growth supplement were seeded in the Matrigel-coated wells and treated with $\operatorname{rhHGF}(3,30 \mathrm{ng} / \mathrm{ml})$ at $37^{\circ} \mathrm{C}$ for $14 \mathrm{~h}$, or co-cultured with CAFs (upper chamber, $1 \times 10^{5}$ cells) supplemented with JNJ-38877605 (400 nM) at $37^{\circ} \mathrm{C}$ for $14 \mathrm{~h}$. Following fixation with $4 \%$ paraformaldehyde at room temperature for $30 \mathrm{~min}$, images of the cells were captured using an inverted phase contrast microscope (Olympus Corporation) at a magnification of x100.

Western blot analysis. CAFs $\left(2 \times 10^{5}\right)$ or NFs in $1 \mathrm{ml} \mathrm{FBS}$-free DMEM supplemented with JNJ-38877605 or not were seeded in the upper chamber of a 6-well Transwell system $(0.4 \mu \mathrm{m}$; Corning), and $2 \times 10^{5}$ HLEC cells in $2 \mathrm{ml} \mathrm{FBS}$-free ECM were seeded in the lower chamber. Following 24 or $48 \mathrm{~h}$ incubations at $37^{\circ} \mathrm{C}$, total proteins were isolated from HLECs using RIPA lysis buffer [50 mM Tris ( $\mathrm{pH} 7.4$ ), $150 \mathrm{mM} \mathrm{NaCl}, 1 \%$ Triton X-100, $1 \%$ sodium deoxycholate, $0.1 \%$ SDS, containing protease and phosphatase cocktails] (Beyotime Institute of Biotechnology, Haimen, China). Protein concentration was determined using a bicinchoninic acid protein assay kit (Beyotime Institute of Biotechnology). Subsequently, $20 \mu \mathrm{g}$ proteins were separated using $8 \%$ SDS-PAGE and electrophoretically transferred onto a nitrocellulose membrane (0.2 $\mu \mathrm{m}$; EMD Millipore, Billerica, MA, USA). Following blocking with 5\% skim milk in Tris-buffered saline and $0.1 \%$ Tween-20 (TBST) for $1 \mathrm{~h}$, membranes were incubated with primary antibodies against c-Met $(1: 2,000)$, phosphorylated (p)-c-Met (1:500) AKT1 (1:5,000), p-AKT1, (1:5,000), $\beta$-tubulin (1:1,000; all from Abcam, Cambridge, UK); PI3K (1:1,000; Tianjin Saierbio Biotechnology, Tianjin, China). Following overnight incubation with primary antibodies 
A

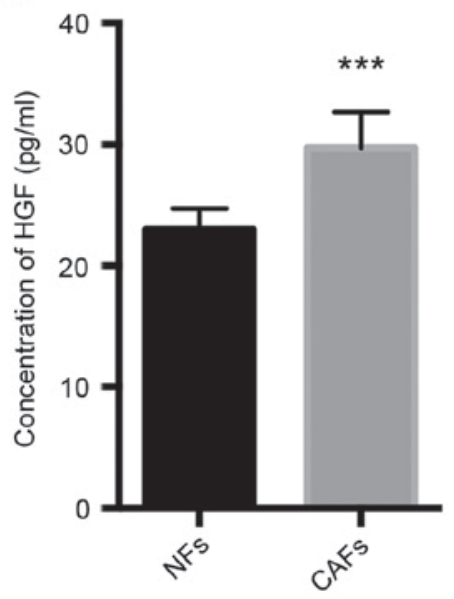

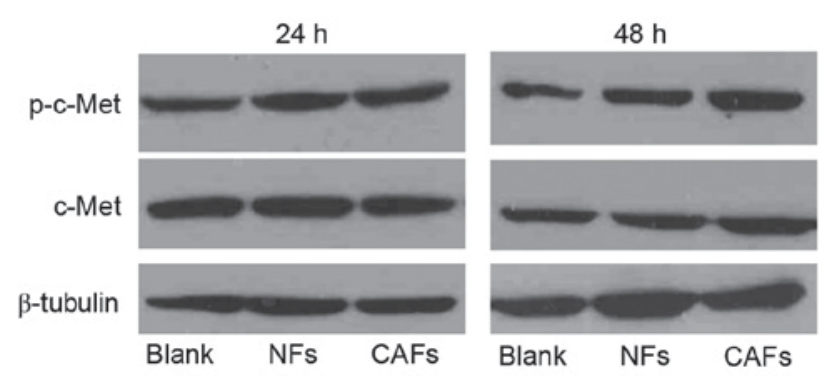

Figure 1. Chemiluminescent immunoassay to determine HGF levels in the supernatant of CAFs and NFs from oral squamous cell carcinoma tissues, and western blots analysis of c-Met and p-c-Met expression in HLECs co-cultured with CAFs and NFs. (A) Representative HGF levels from the supernatant of CAFs and NFs. (B) Representative western blots of c-Met and p-c-Met expression in HLECs co-cultured with CAFs and NFs for 24 and 48 h. ${ }^{* * *}$ P $<0.001$. HGF, hepatocyte growth factor; CAFs, carcinoma-associated fibroblasts; NFs, normal fibroblasts; HLECs, human lymphatic endothelial cells.

diluted in $5 \%$ skim milk in TBST at $4^{\circ} \mathrm{C}$, membranes were incubated with peroxidase-conjugated goat-anti mouse (cat. no. A0216) or goat-anti rabbit (cat. no. A0208) secondary antibodies (1:2,000; Beyotime Institute of Biotechnology) for $1 \mathrm{~h}$ at room temperature. Between each incubation, the membranes were washed 3 times for 5 min each in TBST. Western blots were visualized using Western Lightning Chemiluminescence Reagent (PerkinElmer, Waltham, MA, USA).

Reverse transcription-quantitative polymerase chain reaction (RT-qPCR). Total RNA from HLECs was isolated using TRIzol (Invitrogen; Thermo Fisher Scientific, Inc.) following the manufacturer's protocol. cDNA was synthesized using a PrimeScript RT reagent kit (Takara Bio, Inc., Otsu, Japan). qPCR was performed using a SYBR ${ }^{\circledR}$ Premix Ex gen, Taq ${ }^{\text {TM }}$ II kit (Takara Bio, Inc.) on an ABI 7300 Real-time PCR system (Applied Biosystems; Thermo Fisher Scientific, Inc.). The thermocycling conditions used were as follows: 1 cycle of $95^{\circ} \mathrm{C}$ for $30 \mathrm{sec}, 40$ cycles of $95^{\circ} \mathrm{C}$ for $5 \mathrm{sec}$ and $60^{\circ} \mathrm{C}$ for $31 \mathrm{sec}$, and tubulin was used as a reference gene. The primers for c-Met and $\beta$-tubulin were as follows: c-Met forward, 5'-CATGCCGACAAGTGCAGTA-3' and reverse, 5'-TCT TGCCATCATTGTCCAAC-3'; $\beta$-tubulin forward, 5'-TTG GCCAGATCTTTAGACCAGACAAC-3' and reverse, 5'-CCG TACCACATCCAGGACAGAATC-3'. Data were analyzed using $2^{-\triangle \Delta \mathrm{Cq}}$ method (9). Data were analyzed and compared using the following relative quantification method: $\mathrm{R}=2^{-\Delta \Delta \mathrm{Cq}}$, $\Delta \Delta \mathrm{Cq}=(\mathrm{Cq} 1-\mathrm{Cq} 2)-(\mathrm{Cq} 3-\mathrm{Cq} 4)$, where $\mathrm{R}$ represents the relative amount of the experimental group relative to the NC group; Cq1 represents the cycle threshold of c-Met in the experimental group, $\mathrm{Cq} 2$ represents the cycle threshold of tubulin in the experimental group, $\mathrm{Cq} 3$ represents the cycle threshold of c-Met in the $\mathrm{NC}$ group and $\mathrm{Cq} 4$ represents the cycle threshold of tubulin in the NC group (9).

Statistical analysis. All experiments were conducted in triplicate and representative data are presented as the mean \pm standard deviation. The comparison of treatment effects was evaluated using a two-tailed Student's t-test or one-way ANOVA followed by Holm-Sidak's multiple comparisons test. $\mathrm{P}<0.05$ was considered to indicate a statistically significant difference. Image pro plus 6.0 (Media Cybernetics, Rockville, MD, USA) was used to count the cells in the migration and invasion assays as well as the tubes in the tube formation assay. Statistical analyses were performed using GraphPad Prism 6.0 (GraphPad Software Inc., La Jolla, CA, USA).

\section{Results}

CAFs secrete higher level of HGF than NFs. It is currently unknown whether the contribution of CAFs to lymphangiogenesis in OSCC involves HGF/c-Met. Secreted HGF in the supernatant of CAFs and NFs medium were examined using a CLIA kit. Following $24 \mathrm{~h}$, the secretion of HGF by CAFs $(29.71 \pm 2.963 \mathrm{pg} / \mathrm{ml})$ was significantly higher than the secretion of HGF by NFs $(22.99 \pm 1.717 \mathrm{pg} / \mathrm{ml}$; $\mathrm{P}<0.001$; Fig. $1 \mathrm{~A})$. These data indicate that, compared with NFs, CAFs taken from the TME of OSCC secrete higher levels of HGF.

Overexpression of $p$-c-Met in HLECs co-cultured with CAFs. To evaluate the expression of c-Met and its phosphorylated derivative p-c-Met in HLECs co-cultured with CAFs or NFs, western blot analysis was performed to identify the expression of c-Met and p-c-Met following 24 and $48 \mathrm{~h}$ culture. Co-cultured with CAFs for $48 \mathrm{~h}$, levels of p-c-Met in HLECs were markedly higher than with NFs (Fig. 1B). These data indicate that substances secreted by CAFs promoted the expression of p-c-Met of HLECs. Thus, CAFs may serve an important role in lymphangiogenesis.

HGF promotes the proliferation, migration, invasion and tube formation of HLECs. To determine the regulatory role of HGF on HLECs, HLECs were treated with increasing concentrations of rhHGF and the proliferation, migration, invasion and tube formation of HLECs was subsequently evaluated. A significant dose-dependent difference was observed in the proliferation of HLECs that were treated with rhHGF 
A

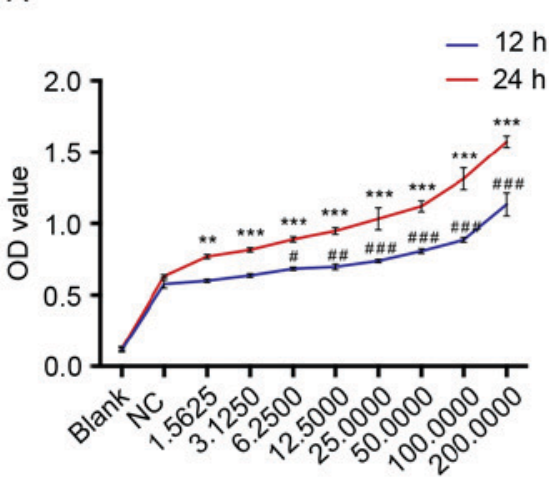

Concentration of HGF $(\mathrm{ng} / \mathrm{ml})$

B

\section{흘}

NC $3 \mathrm{ng} / \mathrm{ml}$ $30 \mathrm{ng} / \mathrm{ml}$

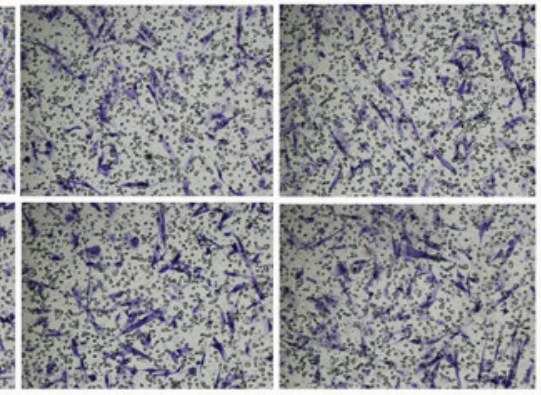

C

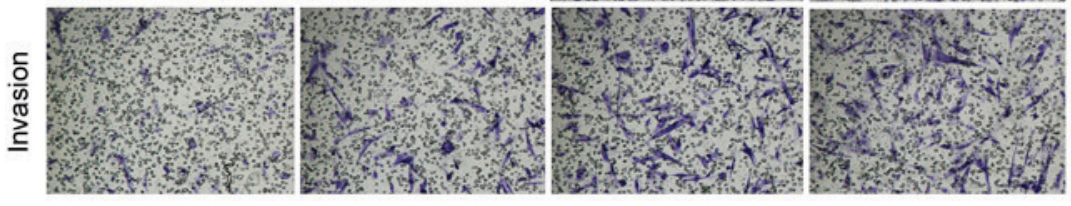

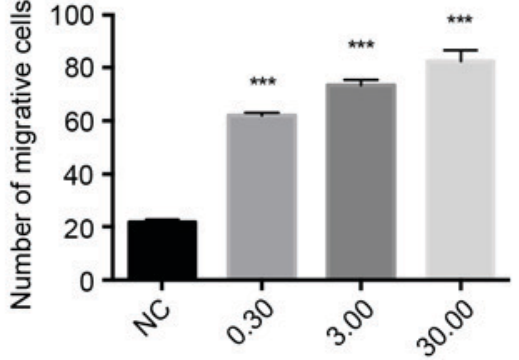

Concentration of HGF $(\mathrm{ng} / \mathrm{ml})$

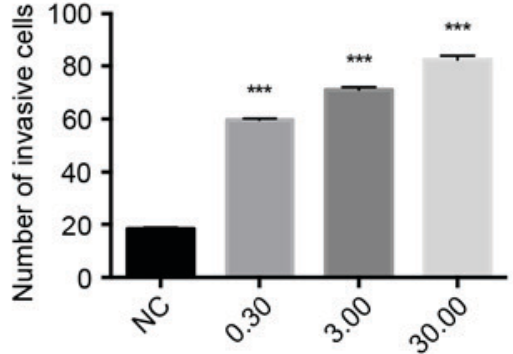

Concentration of $\mathrm{HGF}(\mathrm{ng} / \mathrm{ml})$

Figure 2. rhHGF promotes the proliferation, migration and invasion of HLECs. (A) Cell Counting kit-8 assay for the growth curve of HLECs treated with $1.5625-200 \mathrm{ng} / \mathrm{ml} \mathrm{rhHGF}$ for 12 or $24 \mathrm{~h}$; (B) Images of migrating and invading cells and (C) the number of migrating and invading HLECs treated with 0 , $0.3,3.0$ or $30 \mathrm{ng} / \mathrm{ml} \mathrm{rhHGF}$ for $24 \mathrm{~h}$. Cells were identified using an inverted phase contrast microscope (magnification, x100). Results are the representative of three independent experiments. ${ }^{\#} \mathrm{P}<0.05,{ }^{\# \#} \mathrm{P}<0.01$ and ${ }^{\# \# \#} \mathrm{P}<0.001$ vs. NC after $12 \mathrm{~h} ;{ }^{* *} \mathrm{P}<0.01$ and ${ }^{* * * *} \mathrm{P}<0.001$ vs. NC after $24 \mathrm{~h}$. rhHGF, recombinant human hepatocyte growth factor; HLECs, human lymphatic endothelial cells; NC, negative control; OD, optical density; HGF, hepatocyte growth factor.

A

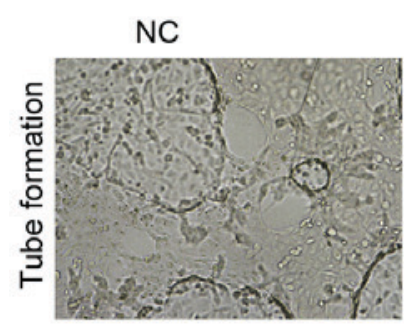

$3 \mathrm{ng} / \mathrm{ml}$

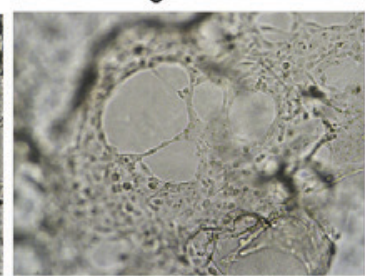

$30 \mathrm{ng} / \mathrm{ml}$

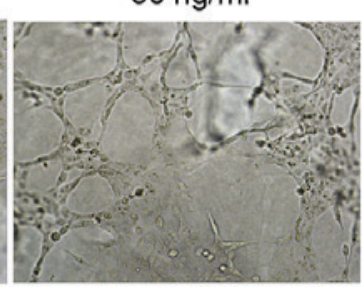

B

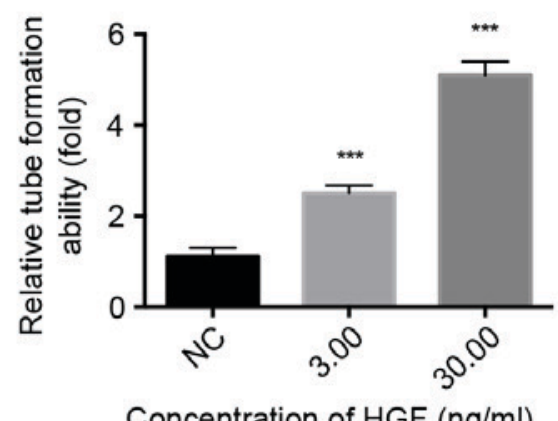

Figure 3. rhHGF promotes the tube formation of HLECs. (A) Matrigel in vitro HLEC tube formation assay of cells treated with $3.0 \mathrm{or} 30 \mathrm{ng} / \mathrm{ml} \mathrm{rhHGF}$ for $14 \mathrm{~h}$. Images were visualized using an inverted phase contrast microscope (magnification, x100). (B) Quantification of tube formation assay. The number of tubes was standardized to relative tube formation ability based on the $\mathrm{NC}$ group. Assays were conducted independently in triplicate. ${ }^{* * *} \mathrm{P}<0.001$. rhHGF, recombinant human hepatocyte growth factor; HLECs, human lymphatic endothelial cells; NC, negative control; HGF, hepatocyte growth factor.

$(1.5625-200 \mathrm{ng} / \mathrm{ml})$ for 12 and $24 \mathrm{~h}$ compared with the $\mathrm{NC}$ (P<0.05; Fig. 2A). The HLECs were induced by $0,0.3,3.0$ and $30 \mathrm{ng} / \mathrm{ml} \mathrm{rhHGF}$. The number of migrating and invading cells significantly increased in a dose-dependent manner following treatment with rhHGF $(\mathrm{P}<0.001$; Fig. $2 \mathrm{~B}$ and $\mathrm{C})$, indicating that rhHGF promotes the migration and invasion of HLECs. Furthermore, following stimulation with 3.0 and $30 \mathrm{ng} / \mathrm{ml}$ rhHGF, HLECs exhibited a significantly higher number of tube formation compared with the NC group ( $\mathrm{P}<0.001$; Fig. 3), indicating that rhHGF is involved in the tube formation of HLECs. Taken together, HGF serves a critical role in modulating the CAFs-associated proliferation, invasion, migration and tube formation of HLECs.
Inhibition of c-Met attenuates the proliferation, migration, invasion and tube formation of HLECs. To know the role of c-Met in lymphangiogenesis, the proliferation, invasion, migration and tube formation of HLECs co-cultured with CAFs and JNJ-38877605 was investigated. The results of the CCK-8 assay indicated that JNJ-38877605 significantly inhibited the proliferation of HLECs compared with the NC group $(\mathrm{P}<0.001$; Fig. 4A). Furthermore, the results of the migration and invasion assays indicated that JNJ-38877605 significantly repressed the migration $(\mathrm{P}<0.01)$ and invasion $(\mathrm{P}<0.001)$ of HLECs compared with the NC group (Fig. 4B-D). JNJ-38877605-treated HLECs formed fewer tubes compared with the NC group $(\mathrm{P}<0.001$; Fig. $4 \mathrm{~B}$ and $\mathrm{E})$. These results 
A

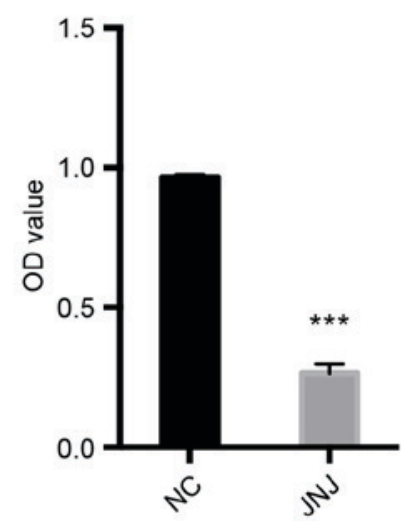

C

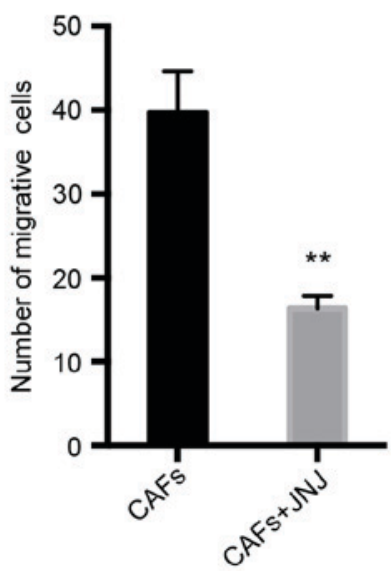

B

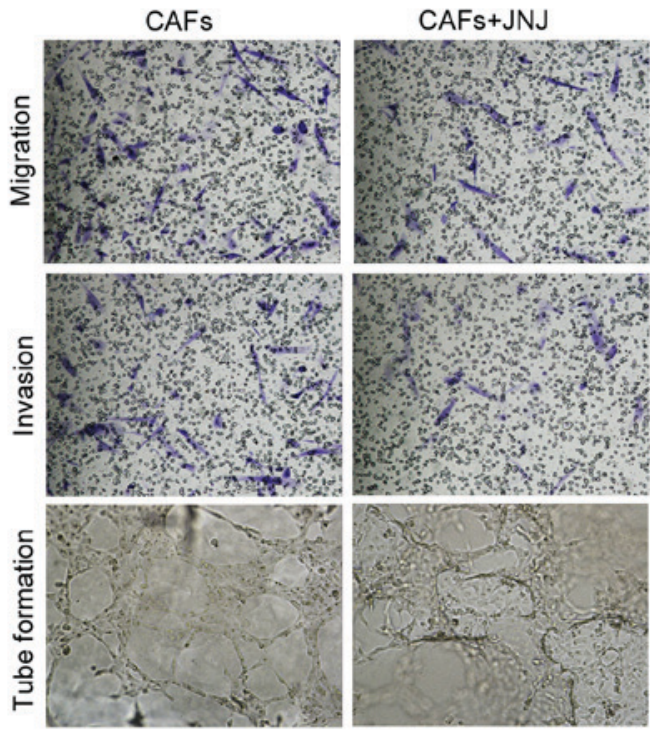

D

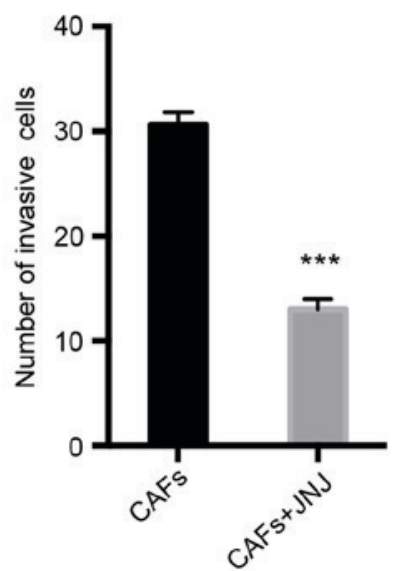

E

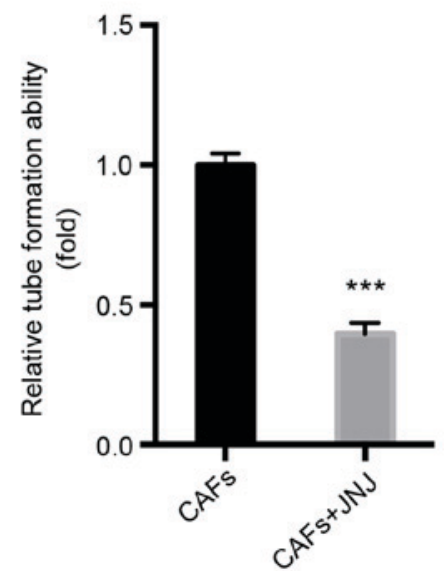

Figure 4. Inhibition of c-Met downregulates the proliferation, migration, invasion and tube formation of HLECs. (A) Cell Counting kit-8 assay measuring the proliferation of HLECs, treated with and without $400 \mathrm{nM} \mathrm{JNJ}$ for $24 \mathrm{~h}$. ${ }^{* * *} \mathrm{P}<0.001$. (B) Images of migrating and invading cells and the tube formation assays. Number of (C) migrating and (D) invading cells. (E) Relative tube formation ability of HLECs treated with and without $400 \mathrm{nM}$ JNJ. Images were visualized using an inverted phase contrast microscope (magnification, $\mathrm{x} 100$ ). All quantitative data were averaged from triple independent results. ${ }^{* *} \mathrm{P}<0.01$ and ${ }^{* * *} \mathrm{P}<0.001$. HLECs, human lymphatic endothelial cells; NC, negative control; CAFs, carcinoma-associated fibroblasts; OD, optical density; JNJ, JNJ-38877605.

indicate that CAFs-modulated $\mathrm{HGF} / \mathrm{c}-\mathrm{Met}$ is crucial for the proliferation, invasion, migration and tube formation of HLECs.

Inhibitor of $c$-Met downregulates the expression of $c$-Met and $P I 3 K / A K T$ in HLECs. To identify the molecules or proteins involved in the signaling pathway, HLECs were co-cultured with CAFs supplemented with or without the c-Met inhibitor, JNJ-38877605. JNJ-38877605 reduced c-Met mRNA compared with the control group $(\mathrm{P}<0.05)$ and markedly downregulated c-Met protein level (Fig. 5A). In addition, western blot analysis indicated that PI3K and p-AKT was also downregulated (Fig. 5B). These data indicate that c-Met/PI3K/AKT may regulate $\mathrm{CAF}$-mediated lymphangiogenesis in the TME of OSCC.

\section{Discussion}

Lymphangiogenesis or invasion by pre-existing lymphatic vessels is the prerequisite of lymphatic metastasis (10). The
TME consists of various secreted soluble cytokines or growth factors, non-cellular solid material, ECM and stromal cells, and all of them surround the tumor cells and support tumor hemangiogenesis, lymphangiogenesis and metastasis (3). Stromal cells, including fibroblasts, smooth muscle, endothelial and inflammatory cells, interact with and modify the TME via the release of growth factors and proteases (11-13). Stromal cells also regulate cell proliferation, apoptosis, invasion and metastasis, as well as hemangiogenesis and lymphangiogenesis (11-13). There is increasing evidence that activated fibroblasts, primarily CAFs, are vital to the TME as they promote tumor growth and hemangiogenesis $(3,14)$. Mantovani et al (15) reported that CAFs are recruited along with hypoxic inducible factor-1 $\alpha$ and promote tumor growth and angiogenesis in the presence of hypoxia release growth factors, including HGF, VEGF and PDGF. It remains unclear whether CAFs contribute to lymphangiogenesis and the mechanism of CAFS in OSCC remains unknown, although certain lymphangiogenic factors have been identified, including fibroblast growth factor, insulin-like growth factor, angiopoietin, 
A

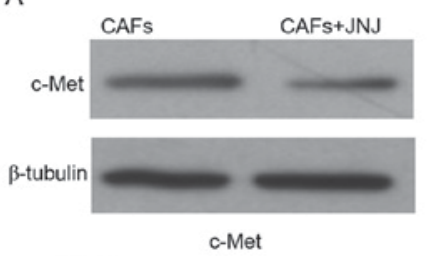

B

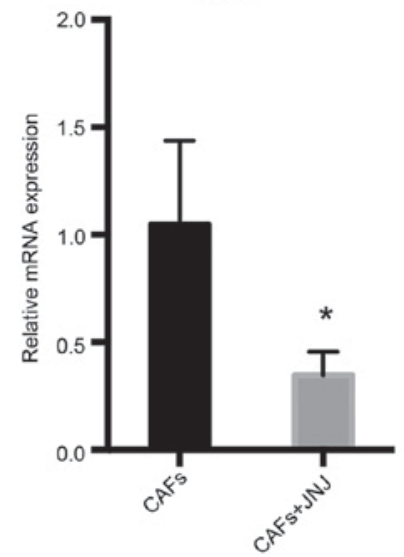

Figure 5. Inhibition of c-Met suppresses the expression of c-Met, PI3K and AKT in HLECs. (A) Reverse transcription-quantitative polymerase chain reaction measuring levels of c-Met mRNA in HLECs co-cultured with CAFs supplemented with or without $400 \mathrm{nM} \mathrm{JNJ}$ for $48 \mathrm{~h}$. (B) Western blot assay of PI3K, p-AKT and T-AKT in HLECs co-cultured with CAFs supplemented with or without $400 \mathrm{nM} \mathrm{JNJ}$ for $48 \mathrm{~h}$. These data are representative of three independent experiments. "P<0.05. PI3K, phosphoinositide 3-kinase; HLECs, human lymphatic endothelial cells; CAFs, carcinoma-associated fibroblasts; JNJ, JNJ-38877605; T-AKT, total protein kinase B; p-AKT, phosphorylated protein kinase $\mathrm{B}$.

VEGF, PDGF and HGF (16). Cao et al (16) successfully induced sprouting and the growth of new lymphatic vessels in a mouse model of corneal lymphangiogenesis via implantation of HGF. In addition, Gibot et al (17) used a human 3D lymphatic vascular construct to identify that HLECs, co-cultured with fibroblasts, spontaneously formed a 3D lymphatic capillary network without the use of any other supplemented factors. It was further demonstrated that fibroblast-derived HGF and VEGF-C work together in lymphangiogenesis by activating the extracellular regulated protein kinase (ERK1/2) signaling pathway (17). Additionally, the roles of $\mathrm{HGF} / \mathrm{c}-$ Met and VEGF-C/VEGF receptor 3 (VEGFR-3) in the formation of lymphatic vessels were identified (17).

The results of the present study revealed that HGF levels were increased in CAFs and that c-Met was overexpressed in HLECs co-cultured with CAFs. Furthermore, it was demonstrated that rhHGF promotes the proliferation, migration, invasion and tube formation of HLECs. A previous study by De Bacco et al (18) determined that ionizing radiation induced the overexpression and activation of the MET oncogene that encodes c-Met via ataxia telangiectasia mutated-nuclear factor- $\kappa \mathrm{B}(\mathrm{NF}-\mathrm{\kappa B})$ signaling. This indicated that MET promotes cell invasion and inhibits apoptosis, facilitating the development of radioresistance. Chen et al (19) demonstrated that JNJ-38877605 decreased the migration of retinal pigment epithelial cells, reduced the phosphorylation of ERK and partially suppressed the activation of protein kinase C (PKC) induced by HGF. These data implied that c-Met, the HGF receptor, contributes to the stimulation of the PKC/ERK signaling pathway. The present study used the co-culture method to simulate crosstalk between CAFs and HLECs in the TME, and the role of $\mathrm{HGF} / \mathrm{c}-\mathrm{Met}$ was subsequently investigated. The results revealed that JNJ-38877605 downregulated the expression of c-Met at the mRNA as well as the protein level.

PI3K, a family of enzymes involved in multiple cell functions, can be phosphorylated and activated by several cell membrane receptor tyrosine kinases, including Met, epidermal growth factor receptors (EGFR), ErbB3 (a type of EGFR), platelet-derived growth factor receptors, VEGFR and insulin-like growth factor 1 receptor (20). PI3K generated by phosphatidylinositol $(4,5)$-bisphosphate, stimulates AKT via translocation to the plasma membrane and subsequently phosphorylates AKT. As a key effector of PI3K, AKT regulates various cellular responses, including the cell cycle, cell proliferation and cell survival, by phosphorylating the relevant proteins (20-22). Yoo et al (23) reported that inhibition of PI3K/AKT blocks sonic hedgehog-induced lymphangiogenesis in gastric cancer. A variety of molecules are involved in the downstream signaling of AKT, including mammalian target of rapamycin, NF- $\mathrm{BB}$, glycogen synthase kinase $3 \beta$, which regulate a varitey of cellular processes including protein synthesis, cell survival and proliferation and make AKT an attravtive therapuetic target for cancer (21). Finally, it was demonstrated that the c-Met inhibitor decreased PI3K and p-AKT and attenuated the proliferation, invasion, migration and tube formation of HLECs co-cultured with CAFs.

In conclusion, our present study preliminarily identified that CAFs serves a vital role on lymphangiogenesis via the Met/PI3K/AKT signaling pathway. Future studies should focus on evaluating the downstream mechanisms, which may help to identify novel potential targets for therapies to inhibit metastasis.

\section{Acknowledgements}

The present study was supported by the Science and Technology Fund of Sichuan Province of China (grant no. 2012JY0075).

\section{References}

1. Roberts TJ, Colevas AD, Hara W, Holsinger FC, Oakley-Girvan I and Divi V: Number of positive nodes is superior to the lymph node ratio and American Joint Committee on Cancer N staging for the prognosis of surgically treated head and neck squamous cell carcinomas. Cancer 122: 1388-1397. 2016.

2. Sipos B, Klapper W, Kruse ML, Kalthoff H, Kerjaschki D and Klöppel G: Expression of lymphangiogenic factors and evidence of intratumoral lymphangiogenesis in pancreatic endocrine tumors. Am J Pathol 165: 1187-1197, 2004.

3. Alphonso A and Alahari SK: Stromal cells and integrins: Conforming to the needs of the tumor microenvironment. Neoplasia 11: 1264-1271, 2009.

4. Fullár A, Kovalszky I, Bitsche M, Romani A, Schartinger VH, Sprinzl GM, Riechelmann H and Dudás J: Tumor cell and carcinoma-associated fibroblast interaction regulates matrix metalloproteinases and their inhibitors in oral squamous cell carcinoma. Exp Cell Res 318: 1517-1527, 2012.

5. Cirri P and Chiarugi P: Cancer-associated-fibroblasts and tumour cells: A diabolic liaison driving cancer progression. Cancer Metastasis Rev 31: 195-208, 2012.

6. Kumar D, Kandl C, Hamilton CD, Shnayder Y, Tsue TT, Kakarala K, Ledgerwood L, Sun XS, Huang HJ, Girod D and Thomas SM: Mitigation of tumor-associated fibroblast-facilitated head and neck cancer progression with anti-hepatocyte growth factor antibody ficlatuzumab. JAMA Otolaryngol Head Neck Surg 141: 1133-1139, 2015. 
7. Sierra JR and Tsao MS: c-MET as a potential therapeutic target and biomarker in cancer. Ther Adv Med Oncol 3 (1 Suppl) S21-S35, 2011

8. Chen S, Gao P, Chang Z and Xuan M: Effects of oral cancer-associated fibroblasts on the proliferation, migration, invasion and tube formation to human lymphatic endothelial cells. Hua Xi Kou Qiang Yi Xue Za Zhi 33: 524-528, 2015 (In Chinese).

9. Livak KJ and Schmittgen TD: Analysis of relative gene expression data using real-time quantitative PCR and the 2(-Delta Delta C(T)) method. Methods 25: 402-408, 2001.

10. Cao Y: Opinion emerging mechanisms of tumour lymphangiogenesis and lymphatic metastasis. Nat Rev Cancer 5: 735-743, 2005.

11. Sund M and Kalluri R: Tumor stroma derived biomarkers in cancer. Cancer Metastasis Rev 28: 177-183, 2009.

12. De Wever O and Mareel M: Role of tissue stroma in cancer cell invasion. J Pathol 200: 429-447, 2003.

13. Pupa SM, Ménard S, Forti S and Tagliabue E: New insights into the role of extracellular matrix during tumor onset and progression. J Cell Physiol 192: 259-267, 2002.

14. Routray S, Sunkavali A and Bari KA: Carcinoma-associated fibroblasts, its implication in head and neck squamous cell carcinoma: A mini review. Oral Dis 20: 246-253, 2014.

15. Mantovani A, Allavena P, Sica A and Balkwill F: Cancer-related inflammation. Nature 454: 436-444, 2008.

16. Cao R, Björndahl MA, Gallego MI, Chen S, Religa P, Hansen AJ and Cao Y: Hepatocyte growth factor is a lymphangiogenic factor with an indirect mechanism of action. Blood 107: 3531-3536, 2006 .
17. Gibot L, Galbraith T, Kloos B, Das S, Lacroix DA, Auger FA and Skobe M: Cell-based approach for 3D reconstruction of lymphatic capillaries in vitro reveals distinct functions of HGF and VEGF-C in lymphangiogenesis. Biomaterials 78: 129-139, 2016.

18. De Bacco F, Luraghi P, Medico E, Reato G, Girolami F, Perera T, Gabriele P, Comoglio PM and Boccaccio C: Induction of MET by ionizing radiation and its role in radioresistance and invasive growth of cancer. J Natl Cancer Inst 103: 645-661, 2011.

19. Chen YJ, Tsai RK, Wu WC, He MS, Kao YH and Wu WS: Enhanced PKC $\delta$ and ERK signaling mediate cell migration of retinal pigment epithelial cells synergistically induced by HGF and EGF. PLoS One 7: e44937, 2012.

20. Simpson DR, Mell LK and Cohen EE: Targeting the PI3K/AKT/mTOR pathway in squamous cell carcinoma of the head and neck. Oral Oncol 51: 291-298, 2015.

21. Liu P, Cheng H, Roberts TM and Zhao JJ: Targeting the phosphoinositide 3-kinase pathway in cancer. Nat Rev Drug Discov 8: 627-644, 2009.

22. Rogers SJ, Box C, Harrington KJ, Nutting C, Rhys-Evans $P$ and Eccles SA: The phosphoinositide 3-kinase signalling pathway as a therapeutic target in squamous cell carcinoma of the head and neck. Expert Opin Ther Targets 9: 769-790, 2005.

23. Yoo YA, Kang MH, Lee HJ, Kim BH, Park JK, Kim HK, Kim JS and Oh SC: Sonic hedgehog pathway promotes metastasis and lymphangiogenesis via activation of Akt, EMT, and MMP-9 pathway in gastric cancer. Cancer Res 71: 7061-7070, 2011.

(1) This work is licensed under a Creative Common Attributis worken under a Creative Commons International (CC BY-NC-ND 4.0) License. 